\title{
SPECTRAL SHAPE SIMULATION USING THE POLYNOMIAL REPRESENTATION
}

\author{
Hana Stefanović ${ }^{1 *}$, \\ Dejan Milić2
}

\author{
${ }^{1}$ School of Electrical and Computer \\ Engineering of Applied Studies, \\ 283, Vojvode Stepe St., Belgrade, Serbia \\ ${ }^{2}$ University of Niš \\ Faculty of Electronic Engineering, \\ Niš, Serbia
}

\begin{abstract}
:
In this paper, the spectral shape is estimated using polynomial model based on ARMAX structure (Autoregressive moving-average model with exogenous input). The stochastic signal with obtained power spectral density is generated in MATLAB, while the periodogram method for estimating the spectral density based on signal samples is used. The simulation of simple wireless system in which the motion is present is also given, based on generation of a process having a power spectral density representing the Doppler effect.
\end{abstract}

Key words:

ARMAX model, doppler effect, polynomial model, wireless system simulation.

\section{INTRODUCTION}

A polynomial model uses a generalized notion of transfer functions to express the relationship between the input $u(t)$, the output $y(t)$, and the noise $e(t)$ using the equation (Kovacevic and Djurovic, 1999):

$$
H(z)=B\left(z^{-1}\right) / A\left(z^{-1}\right)
$$

where $H(z)$ presents a pulse transfer function, $A(z)$ and $B(z)$ are polynomials in $z$ or $z^{-1}$, while $z^{-1}$ is the backward shift operator, or time-shift operator, stating that $z^{-1} y(k)=y(k-1) . A(z)$ and $B(z)$ are polynomials with respect to the backward shift operator, defined by (Golnaraghi and Kuo, 2009):

$$
A\left(z^{-1}\right)=1+\sum_{i=1}^{n} a_{i} z^{-i}
$$

and

$$
B\left(z^{-1}\right)=\sum_{i=1}^{m} b_{i} z^{-i}
$$

Hana Stefanović

e-mail:

stefanovic.hana@yahoo.com
Equivalent time domain representation, relating the output sequence $\{y(k)\}$ to the input sequence $\{u(k)\}$ is given by: 


$$
y(k)=\frac{B\left(z^{-1}\right)}{A\left(z^{-1}\right)} u(k)=\frac{\sum_{i=1}^{m} b_{i} z^{-i}}{1+\sum_{i=1}^{n} a_{i} z^{-i}} u(k)
$$

It can therefore be concluded:

$$
y(k)-\sum_{i=1}^{n} a_{i} y(k-i)=\sum_{i=0}^{m} b_{i} u(k-i)
$$

When the system is excited by a random input sequence $\{e(k)\}$ and a deterministic input sequence socalled exogenous input $\{u(k)\}$, the model is written in the following form:

$$
A\left(z^{-1}\right) y(k)=B\left(z^{-1}\right) u(k)+C\left(z^{-1}\right) e(k)
$$

where polynomial $C(z)$ is given with:

$$
C\left(z^{-1}\right)=1+\sum_{i=0}^{l} a_{i} z^{-i}
$$

This model is known in the literature as the autoregressive moving-average model with exogenous input, or ARMAX model. The ARMAX model represents the general form for popular time-series and digital filter models:

- for $C=0$, the pulse transfer function or infinite impulse response (IIR) model

- for $A=1$ and $C=0$, the finite impulse response (FIR) model

- for $B=0$ and $C=1$, the autoregressive (AR) model

- for $A=1$ and $B=0$, the moving-average (MA) model

- for $B=0$, the autoregressive moving-average (ARMA) model

- for $C=1$, the autoregressive model with exogenous input (ARX)

The ARMAX model is usually used for signal processing purposes (Red-Horse et al., 1996), since the timeseries analysis based on ARMAX technique has many advantages over the traditional spectral method (Peng et al., 2001).

\section{MODELING OF STOCHASTIC PROCESS}

A random sequence with autocovariance:

$$
R_{Y}(n T)=a^{|n T|}, \quad 0<a<1, T=1
$$

is proposed. The power spectrum is:

$$
\begin{aligned}
S_{Y}(z) & =\phi\left\{R_{Y}(n)\right\}=\sum_{n=-\infty}^{+\infty} R_{Y}(n) z^{-n} \\
& =S_{Y}^{+}(z)+S_{Y}^{-}(z)-R_{Y}(0)
\end{aligned}
$$
time:

with $S_{Y}^{+}$and $S_{Y}^{-}$signifying positive and negative

$S_{Y}^{+}(z)=\sum_{n=0}^{+\infty} R_{Y}(n) z^{-n}=\sum_{n=0}^{+\infty}\left(a z^{-1}\right)^{n}=\frac{1}{1-a z^{-1}}$

and:

$$
S_{Y}^{-}(z)=\sum_{n=-\infty}^{0} R_{Y}(n) z^{-n}=\sum_{n=-\infty}^{0}(a z)^{-n}=\frac{1}{1-a z}
$$

It can be concluded (Kovacevic and Djurovic, 1999):

$$
\begin{aligned}
S_{Y}(z) & =\frac{1}{1-a z^{-1}}+\frac{1}{1-a z}-1=\frac{1-a^{2}}{\left(1-a z^{-1}\right)(1-a z)} \\
& =H(z) H\left(z^{-1}\right) \sigma_{u}^{2}=H(z) H\left(z^{-1}\right)\left(1-a^{2}\right)
\end{aligned}
$$

Since $S_{u}(z)=\sigma_{u}^{2}=1-a^{2}$, transfer function $H(z)$ is:

$$
H(z)=\frac{1}{1-a z^{-1}}
$$

By using the autoregressive model representation, it can be written:

$$
H(z)=\frac{\phi\{y(k)\}}{\phi\{e(k)\}}=\frac{Y(z)}{E(z)}=\frac{1}{1-a z^{-1}}
$$

with the difference equation obtained:

$$
y(k)=a y(k-1)+e(k)
$$




\section{GENERATING THE STOCHASTIC SIGNAL WITH OBTAINED POWER SPECTRAL DENSITY}

The stochastic signal with the obtained power spectrum is generated in MATLAB, using the periodogram method for estimating the spectral density based on the signal samples (Hayes, 1996). Periodogram is given by the modulus squared of the Discrete Fourier Transform (DFT) and is one of the simplest techniques to estimate the spectrum (Therrien, 1992).

Some numerical examples for different values of parameter $a$ are given in Fig. 1, Fig. 2 and Fig. 3. The theoretical and estimated power spectral density is shown.

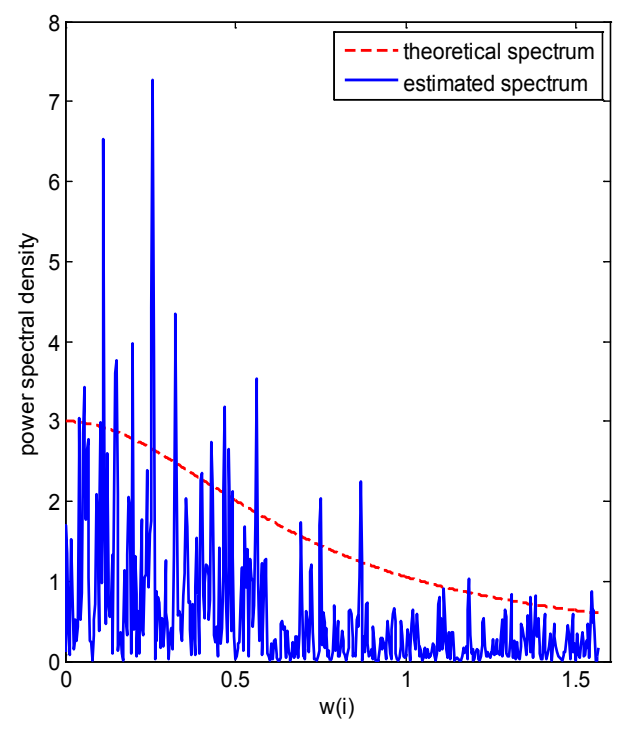

Figure 1. Power spectral density for $a=0.5$

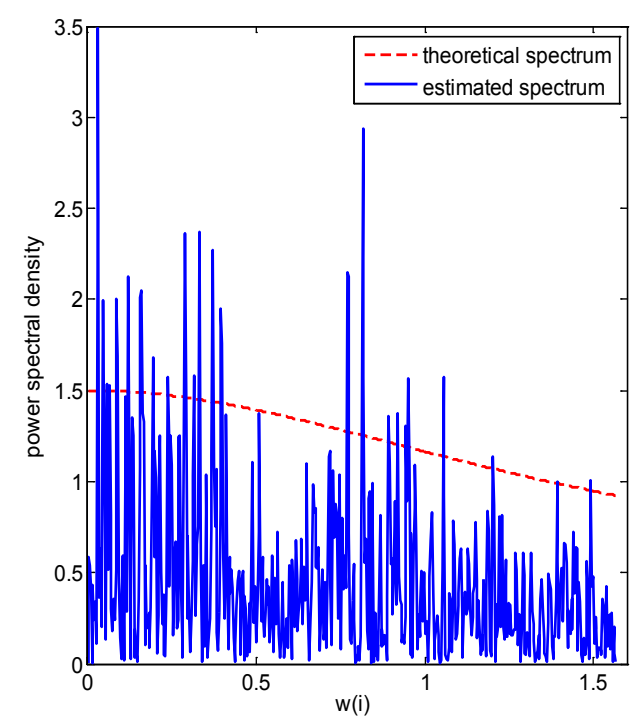

Figure 2. Power spectral density for $a=0.2$

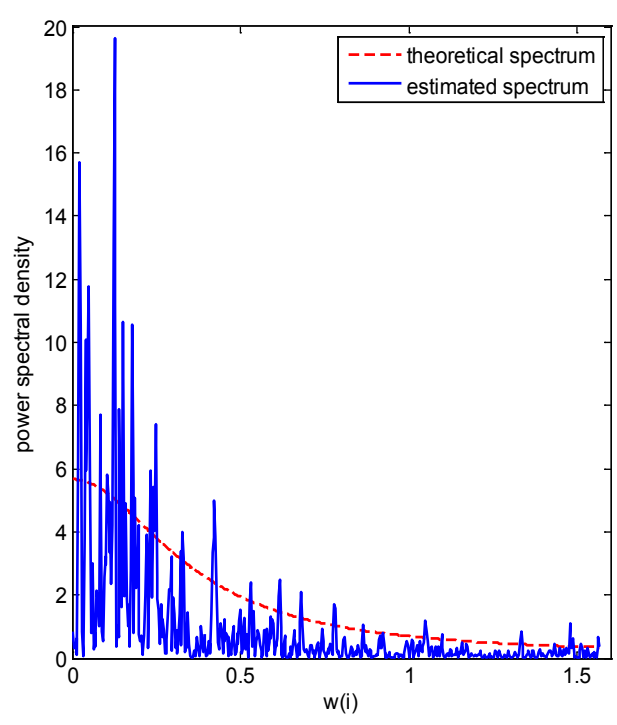

Figure 3. Power spectral density for $a=0.7$

\section{THE SIMULATION OF WIRELESS SYSTEM WITH GIVEN POWER SPECTRAL DENSITY}

A wireless communication system providing access to the communication infrastructure for mobile users is proposed. The presence of multiple paths between the transmitter and receiver is due to reflection and refraction. Multipath and motion-induced fading is one of the most severe performance-limiting phenomena that occur in wireless mobile communication channels (Proakis and Salehi, 2008).

In this paper, the power spectral density model assumes that there are many multipath components, each having different delays, while all components have the same Doppler spectrum. Each component is made up of a sum of simultaneously arriving unresolvable paths, having angle of arrival with a uniform angular distribution at the receiver side, like in Jakes model (Tranter et al., 2004). The classical Jakes' Doppler spectrum has a form (Jakes, 1974):

$$
S(f)=\frac{K}{\sqrt{1-\left(f / f_{d}\right)^{2}}},|f| \leq f_{d}
$$

where $f_{d}=v / \lambda$ is the maximum Doppler shift, $v$ is the vehicle speed and $\lambda$ is the wavelength of the carrier. The Doppler spectrum defined by (16) is appropriate for dense scattering environments like urban areas. Typical Doppler bandwidths in mobile applications at $1 \mathrm{GHz}$ will range from 10 to $200 \mathrm{~Hz}$.

It is supposed that the noise samples are independent, so power spectral density (PSD) of system output $S_{Y}(f)$ is: 


$$
S_{Y}(f)=|H(f)|^{2} S_{X}(f)
$$

Since the PSD of the input is constant, it can be written:

$$
S_{Y}(f)=|H(f)|^{2} K
$$

The required transfer function to establish the target power spectral density is:

$$
H(f)=\sqrt{S_{Y}(f) / K}
$$

or equivalently (Tranter et al., 2004):

$$
H(f)= \begin{cases}\left(1-\left(f / f_{d}\right)^{2}\right)^{-1 / 4}, & |f| \leq f_{d} \\ 0, & \text { otherwise }\end{cases}
$$

This filter is implemented in MATLAB as a FIR filter whose impulse response is obtained by taking the inverse DFT of sampled values of $H(f)$. Therefore, the problem of shaping the power spectral density to meet the given requirements reduces to the problem of finding a filter with a transfer function $H(f)$ so $H^{2}(f)$ gives the required spectral shape.

The impulse response and transfer function are illustrated in Fig. 4 and Fig. 5, respectively, using Jakes' model.

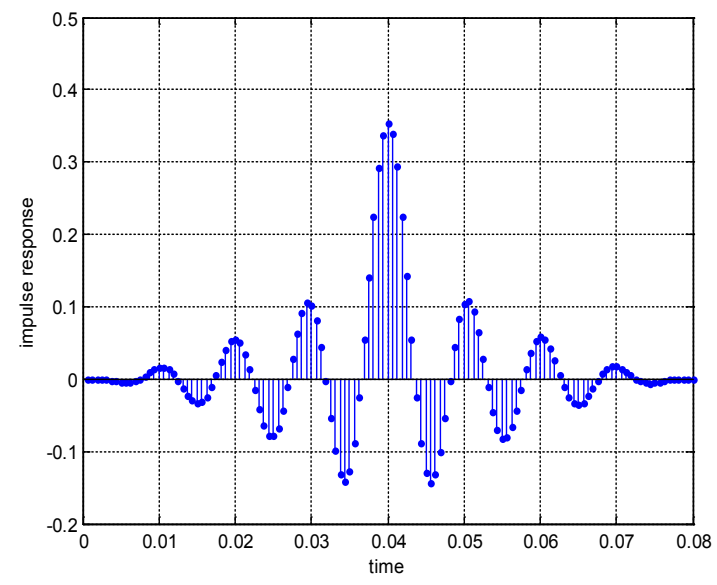

Figure 4. The impulse response

The effect of filtering a complex white noise is illustrated in Fig.6. and Fig.7.

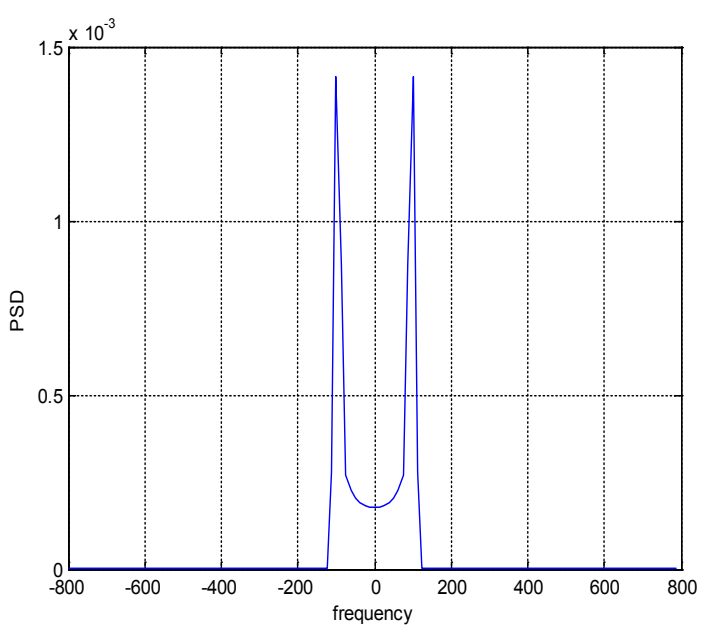

Fig. 5. Power spectral density

The estimated PSD at the filter output is given in Fig.6, while Fig. 7. illustrates the magnitude of the envelope, corresponding to the fading effect in mobile communications.

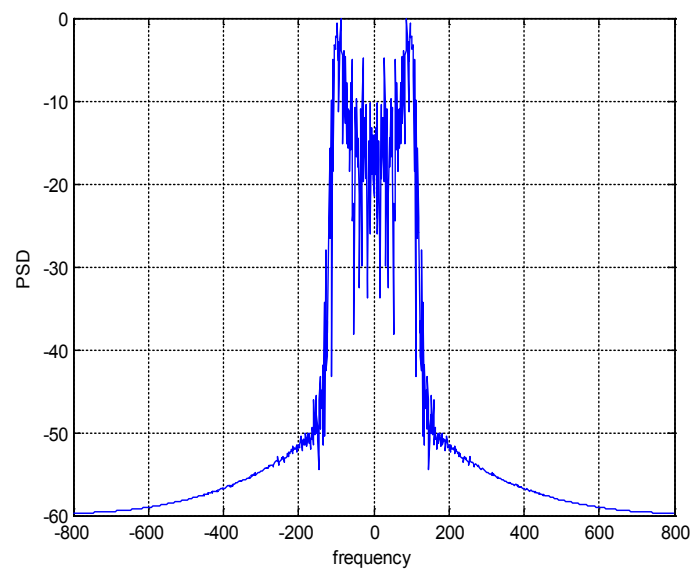

Figure 6. Power spectral density at the filter output

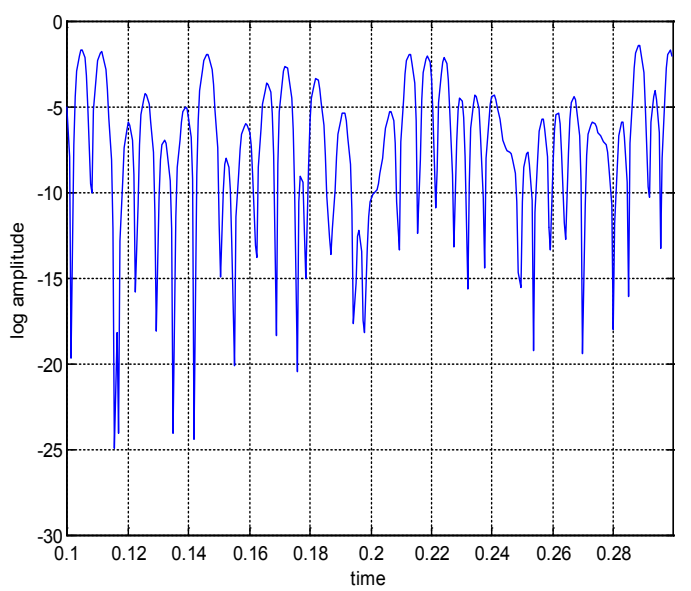

Figure 7. The the magnitude of fading envelope 


\section{CONCLUSION}

In this paper, the distribution of the power over frequencies for different realizations of a random signal, generated by the same ARMAX model, using different realizations of the noise and different model coefficients, is analyzed. The power spectral density and an estimate of the power spectral density obtained from a short realization of the random process, are also presented, describing the fading effect in mobile wireless communications.

\section{REFERENCES}

Golnaraghi, F., \& Kuo B.C. (2009). Automatic Control Systems (9th ed.). Hoboken: John Wiley \& Sons.

Hayes, M.H. (1996). Statistical Digital Signal Processing and Modeling. New York: John Wiley \& Sons.

Jakes, W.C. (1974). Microwave Mobile Communications. New York: John Wiley \& Sons.
Kovacevic, B., \& Djurovic, Z. (1999). Fundamentals of Stochastic Signals, Systems and estimation Theory with Worked Examples. Belgrade: Academic Mind.

Peng, H., Ozaki, T., Toyoda Y., \& Oda K. Modeling and control of systems with signal dependent nonlinear dynamics. In Proceedings of the European Control Conference, 2001 (42-47). Porto, Portugal.

Proakis, J., \& Salehi M. (2008). Digital Communications. (5th ed.). Boston: McGraw-Hill.

Red-Horse, J.R., Alvin, K.F., Mingolet, M.P., \& Robertson, A.N. An investigation of three major timeseries data analysis techniques. In IMAC XIV-14th International Modal Analysis Conference, 1996 (1600-1607). Michigan, USA.

Therrien, C.W. (1992). Discrete Random Signals and Statistical Signal Processing. Englewood Cliffs, N.J.: Prentice-Hall.

Tranter, W., Shanmugan, K., Rappaport, T., \& Kosbar, K. (2004). Communication Systems Simulation with Wireless Applications, NJ: Prentice Hall. 